first step of surgery of this lesion, if the brain is kept off from irreversible ischemic damage.

\title{
f-11. Application of Huge Electrical Magnet for Artificial Embolization
}

\author{
Kenichiro Sugita, Terushige Dor, Yoshiro TaKaoka, Naomi Mutsuga, \\ Ryuichi Tsugane and Osamu Sato \\ Ist Department of Surgery, Nagoya University School of Medicine
}

Since the report of A.J. Luessenhop, the method of artificial embolization has been improved in several aspects, however, it is still problematical how to introduce the emboli to the selected vessels and it is also the matter of debate how to leave or keep the material at the initially introduced sites. We have experienced a case on which introduction of the steel balls into the feeding vessels of a tumor by means of extracranially placed magnetic device was applied.

Magnetic field of the electrical magnet made by Schumann was 7,000 Gauss at the distance of $1 \mathrm{~cm}$ from the tip and 500 Gauss at $10 \mathrm{~cm}$. In the experiments with water flow in the $\mathrm{T}$ or $\mathrm{Y}$ shaped glass tube models, the maximum distance between the bifurcation of glass tube and the tip of magnet was measured at various water pressures, at which the steel ball could be controlled to flow into the aimed direction. The relationship between the distance and the water pressure was as follows; $5 \mathrm{~cm}$ at $150 \mathrm{mmHg}, 7 \mathrm{~cm}$ at $80 \mathrm{mmHg}$ and $9 \mathrm{~cm}$ at $50 \mathrm{mmHg}$. The inner diameter of glass tube $(5-2 \mathrm{~mm})$ an dthe diameter of steel ball $(3.0-$ $0.8 \mathrm{~mm}$ ) had practically no influence on distance-pressure relationship.

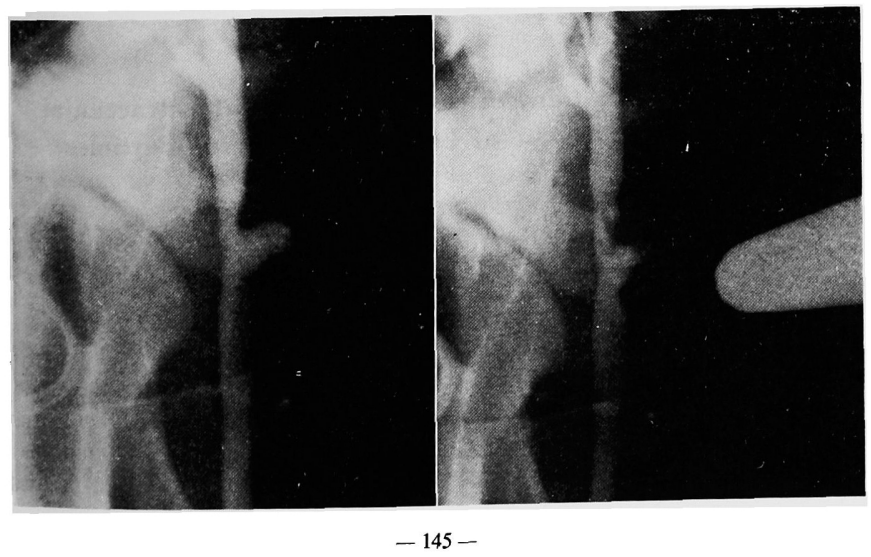


Then we performed animal experiment using aorta of the dog. Steel ball emboli were introduced into the aorta from common carotid artery and the emboli were controlled to flow into superior mesenteric artery, right and left renal arteries using electrical magnet placed $5-7 \mathrm{~cm}$ apart from the origin of these arteries. The results were completly satisfactory.

The case presented herein had been aphasic and suffered from right hemiplegia for years harbouring a large parasagittal meningioma on the left and operated upon two times when only partial removals were performed because of extreme hemorrhage. Marked vascularization was noticed which was mainly contributed from the left anterior cerebral artery and this was confirmed by means of serial angiography. The ratio of calibers of the anterior and middle cerebral arteries. was five to four at the bifurication with the anterior one directing acutely toward right. At the time of artificial embolization the magnetic device was snuggly attached to the right anterior temporal as the distance was measured $9 \mathrm{~cm}$ from the magnet to the left bifurcation. Under local anesthesia the left external carotid artery was exposed from which two steel balls with the diameter of $0.8 \mathrm{~mm}$, twenty of $1.5 \mathrm{~mm}$ and five of $2.0 \mathrm{~mm}$ diameter were introduced into the internal carotid artery. Initially small ones and then caliber was increased. Seven out of twenty-seven steel balls were mislead into the middle cerebral artery and its branches, however, the patient became gradually alert during the procedure being able to give us his name and address properly. A large tumor weighing $420 \mathrm{Gm}$ was successfully extripated three weeks following the above stated procedure and the patient did well after the craniotomy.

With the use of huge electrical magnet and the steel balls as emboli, artificial embolization may have wider therapeutic application in the treatment of inoperable A-V malformations and aneurysms and vascular tumors.

\title{
Discussion to $\mathrm{f}-11$.
}

\section{The Magnetically Controlled Thrombosis of Intracranial Aneurysms by the Use of Ferromagnetic Fine Particles}

\author{
Yasuaki Yoshida, Kozo Yoshida, Shinsuke Tominaga, \\ Kazuo Hashi and Hajime HaNda \\ Department of Neurosurgery, Kyoto University School of Medicine
}

Suggested by the study of Alksne et al., we have also been studying magnetically controlled thrombosis of intracranial aneurysms by the use of ferromagnetic fine particles. Our intention to use this method is only to those cases in which neck clipping, träpping or coating of the aneurysm is found to be difficult when intracranial direct attack is performed. Therefore, it is necessary 